\title{
Small RNA profiles in soybean primary root tips under water deficit
}

\author{
Yun Zheng', Vandana Hivrale ${ }^{2}$, Xiaotuo Zhang ${ }^{1}$, Babu Valliyodan ${ }^{3}$, Christine Lelandais-Brière $^{4,5}$, Andrew D. Farmer ${ }^{6}$, \\ Gregory D. May ${ }^{6,7}$, Martin Crespi ${ }^{4,5}$, Henry T. Nguyen ${ }^{3^{*}}$ and Ramanjulu Sunkar ${ }^{2^{*}}$
}

From 15th International Conference On Bioinformatics (INCOB 2016)

Queenstown, Singapore. 21-23 September 2016

\begin{abstract}
Background: Soybean (Glycine max) production is significantly hampered by frequent droughts in many regions of the world including the United States. Identifying microRNA (miRNA)-controlled posttranscriptional gene regulation under drought will enhance our understanding of molecular basis of drought tolerance in this important cash crop. Indeed, miRNA profiles in soybean exposed to drought were studied but not from the primary root tips, which is not only a main zone of water uptake but also critical for water stress sensing and signaling.

Methods: Here we report miRNA profiles specifically from well-watered and water-stressed primary root tips (0 to $8 \mathrm{~mm}$ from the root apex) of soybean. Small RNA sequencing confirmed the expression of vastly diverse miRNA (303 individual miRNAs) population, and, importantly several conserved miRNAs were abundantly expressed in primary root tips.

Results: Notably, 12 highly conserved miRNA families were differentially regulated in response to water-deficit; six were upregulated while six others were downregulated at least by one fold (log2) change. Differentially regulated soybean miRNAs are targeting genes include auxin response factors, Cu/Zn Superoxide dismutases, laccases and plantacyanin and several others.

Conclusions: These results highlighted the importance of miRNAs in primary root tips both under control and waterdeficit conditions; under control conditions, miRNAs could be important for cell division, cell elongation and maintenance of the root apical meristem activity including quiescent centre whereas under water stress differentially regulated miRNAs could decrease auxin signaling and oxidative stress as well as other metabolic processes that save energy and water.
\end{abstract}

Keywords: Drought, miRNAs, Primary root tips, Post-transcriptional gene regulation, Soybean

\section{Background}

MicroRNAs are known to regulate the abundances of messenger RNAs that have complete or partial complementarity and inhibit protein synthesis from those targets [1, 2]. MicroRNA-guided gene regulation is vital for almost all aspects of plant growth and development [2-4] as well as biotic and abiotic stress responses [5].

\footnotetext{
*Correspondence: NguyenHenry@missouri.edu;

ramanjulu.sunkar@okstate.edu

${ }^{3}$ National Center for Soybean Biotechnology and Division of Plant Sciences,

University of Missouri, Columbia, MO 65211, USA

${ }^{2}$ Department of Biochemistry and Molecular Biology, Oklahoma State

University, Stillwater, OK 74078, USA

Full list of author information is available at the end of the article
}

Soybean (Glycine max) is a major leguminous crop cultivated for vegetable oil and protein source for human consumption and animal feed, besides being used in aquaculture and poultry [6]. In the recent years, drought has significantly affected soybean yields throughout the world including the United States. Root system is most important organ with respect to drought responses; therefore, analyzing miRNAs could reveal critical processes important for drought tolerance in soybean. Within the root system, well-developed or older or matured root zone is less active not only metabolically but also with respect to its role in signalling, therefore analyzing primary root tip is critically important not only 
under water deficit conditions but also under normal conditions.

A recent study profiled small RNAs from diverse soybean tissues [7] but not from the primary root tips. Similarly, miRNAs were profiled from the entire root system of soybean exposed to drought or water stress, but not in the primary root tips $[8,9]$. These published studies found similar as well as distinct regulations with respect to the responsiveness of various miRNAs, which could be attributed to differences in genotypes, treatments, duration or other experimental variables.

Compared to many soybean genotypes, the soybean cv. Magellan, which was used in the current study, was relatively drought-tolerant $[10,11]$. Interestingly, on the basis of differential responses with respect to elongation rates during stress, primary root tip region can be divided into two regions (Region $1[0-4 \mathrm{~mm}$ from the apex, including the root cap] and Region 2 [4-8 mm from the apex]) in this genotype [10]. Under water stress, Region 1 maintains elongation rates but Region 2 shows progressive deceleration, however, under wellwatered conditions, only Region 2 maintains maximum elongation rates $[10,11]$. Well-developed or older or matured root zone is less active not only metabolically but also with respect to its role in signalling, therefore analyzing molecular responses in primary root tip is critically important under water deficit conditions. Previously, proteome [10] as well as transcriptome [12] changes in response to drought have been analyzed in soybean primary root tips. However, post-transcriptional gene regulation directed by miRNAs in this region of the soybean roots has not been investigated so far. Studying miRNA-guided gene regulations is a rational link that could help bridge the gap between transcriptome and proteome analysis in this important organ of the plant system. Therefore, here we report miRNA profiles to further our understanding of drought tolerance mechanisms in the primary root tips of a tolerant soybean genotype.

\section{Methods}

\section{Stress treatments, collection of root tips, RNA isolations} and sequencing

The soybean seedlings (Magellan genotype) growth and treatments were conducted as reported previously [10]. In brief, seedlings with primary roots approximately $15 \mathrm{~mm}$ in length were transplanted against the interior surface of Plexiglas cylinders $(14.5 \mathrm{~cm}$ diameter $)$ filled with a 1:1 (v/v) mixture of vermiculite (no. 2A, ThermORock East Inc., New Eagle, PA, USA) and Turface (Profile Products LLC, Buffalo Grove, IL, USA) at water potentials of $-0.1 \mathrm{MPa}$ (well-watered control, moistened to the drip point) or $-1.6 \mathrm{MPa}$ (water stress), which were obtained by thorough mixing with different volumes of
$5 \mathrm{~mm} \mathrm{CaCl} 2+5 \mathrm{~mm} \mathrm{Ca}\left(\mathrm{NO}_{3}\right)_{2}$ solution. Primary root tip region $(0-8 \mathrm{~mm})$ was collected after $48 \mathrm{~h}$ of treatment along with the control samples in two replicates and total RNA was isolated and used for constructing small RNA libraries. Root tip water stress level was assessed by measuring water potential of the growing media using an isopiestic thermocouple psychrometer. The obtained small RNA libraries were sequenced using Illumina HiSeq 2000 equipments by following the corresponding protocols. Two replicate libraries per treatment (control and water-stressed) were constructed and sequenced. The obtained small RNA profiles had been deposited into NCBI SRA database under the series accession number SRP083572.

\section{Sequence analysis, identification of conserved and novel miRNAs as well as drought-responsive miRNAs}

The extracted small RNA reads were screened against ribosomal and transfer RNAs, snRNAs and snoRNAs and exact matches to these RNA categories were discarded. Similarly, reads mapped to the transposons and protein-coding mRNAs were also discarded. Also that small RNAs shorter than 18 nucleotide (nt) and longer than 28 nt were discarded. The remaining reads were mapped to the miRBase to identify conserved and known miRNA homologs. The reads that could not belong to any of the above mentioned RNA categories were mapped to the genome in order to identify potential novel miRNAs, whose identification strictly followed the guidelines of Meyers et al. [13]. In brief, we mapped novel small RNAs to the genome and isolated the precursor region, which is about $150 \mathrm{nt}$ both downstream and upstream to the mapped region and predicted foldback structure for the precursor. If a fold-back structure could be predicted for the precursor region, then we searched for the presence of miRNA* reads in our small RNA libraries that is complementary to the potential novel miRNA.

For calculating the raw abundance of individual miRNAs within a family, the unique reads were used. Similarly, the raw abundance of all members were pooled to count the abundances of miRNA families. Abundances for each miRNA/miRNA family were normalized to Reads per 10 million (RPTM). For identification of drought-responsive miRNAs, fold $(\log 2)$ change values were calculated. edgeR [14] was used to identify differentially regulated miRNAs and miRNA families in the treated primary root tips.

\section{Target prediction of miRNAs and tasiRNAs}

The targets of miRNAs and tasiRNAs were predicted using the HitSensor algorithm [15]. Targets with less than 4 mismatches were kept for further analysis. The 
enrichment analysis for targets of some highly expressed deregulated miRNAs were performed with agriGO [16].

\section{Results \\ Deep sequencing of small RNAs from soybean root tips exposed to drought}

Two replicate small RNA libraries were constructed and sequenced from primary root tips of control and water deficit-exposed soybean seedlings. Each of these libraries (control and treated) from primary root tips yielded approximately 2 million reads. Overall, 7.5 million total reads represented by 1.8 million unique reads were obtained from these four small RNA libraries from primary root tips (Table 1). Small RNA length distribution versus abundances revealed a peak each at 21 and 24 nt size classes, resembling typical plant small RNA populations (Fig. 1). On the basis of their sequence similarity with the deposited miRNAs at the miRBase, we have identified 303 miRNA homologs (Additional file 1), which could be clustered into 171 families (Additional file 2).

\section{Analysis of miRNA abundances in primary root tips of soybean}

MicroRNAs have emerged as critical regulators of gene expression which is important for normal growth and development of leaves, roots, flowers and other morphological traits in plants. Primary root apex is critical for root growth and development under normal conditions. To assess the importance of miRNAs in this root zone of soybean, we measured their levels. Indeed, the abundances of different miRNA families varied greatly in the primary root tips. On the basis of their normalized abundances, miRNA families were divided into high (100,000 RPTM and above), moderate $(10,000$ to 100,000 RPTM), low (1000 to 10,000 RPTM) and extremely low (1 to 1000 RPTM) abundant families. Of the 171 miRNA families, five (miR156, miR166, miR1507, miR1509 and miR1510) were categorized as highly abundant. Of these, miR1507 is the most abundant in primary root tips as its expression levels were as high as almost 800,000 RPTM accounting for almost onetwelfth of the total small RNAs sequenced in each of the four libraries. A very high abundance of these 5 miRNA families in this region indicates important gene regulatory roles for these miRNAs in primary root tips. miR156 regulates several SBP transcription factors and miR166 targets HD-ZIP transcription factors whereas miR1507, miR1509 and miR1510 largely found in legumes so far.

Six miRNA families (miR168, miR396, miR1511, miR1508, miR172 and miR3522) were grouped as moderately abundant in primary root tips (Additional file 2). The miR168 family is the most enriched in this group followed by miR396 and miR1511 families. miR168 regulates Argonaute-1, an essential protein for miRNA activity; miR396 regulates several GRF transcription factors that are mostly known to regulate leaf development [17] but also central for normal root growth and development [18] and miR1508 and miR1511 appears to be mostly found in legumes thus far, suggesting lineagespecific roles. miR1507, miR 1508, miR 1509, and miR 1510 , are involved in triggering 21 nucleotide phasiRNA biogenesis in legumes [19]. Nineteen miRNA families (miR5767, miR4345, miR169, miR2109, miR482, miR2119, miR2118, miR171, miR164, miR4413, miR390, miR1514, miR159, miR160, miR5781, miR167, miR5373 and miR4409) displayed lower abundances (1000 to 10,000 RPTM) (Additional file 2). This group included a large number of conserved miRNA families, which are known to regulate diverse families of transcription factors such as NF-Y, Scarecrow-like, NAC, MYB, and ARFs. These targets have wide-range of roles regulating diverse developmental as well as other processes in plants [2-4]). Approximately 33 miRNA families (miR1516, miR1517, miR1519, miR1531, miR2107, miR395, miR399 miR4340, miR4350, miR4356, miR4358, miR4368, miR4372, miR4375, miR4382, miR4396, miR4399, miR4401, miR4402, miR4405, miR4414, miR4995, miR5035, miR5369, miR5379, miR5669, miR5673, miR5675, miR5676, miR5679, miR5766, miR5772 and miR862) were extremely low abundant (less than 10 RPTM) in the primary root tips. The extreme low abundance of these miRNAs is in agreement with Arikit et al. [7], who classified these as siRNA-like

Table 1 Summary of small RNA reads obtained from the primary root tips exposed to water deficit or control plants

\begin{tabular}{|c|c|c|c|c|c|c|c|c|}
\hline & \multicolumn{2}{|l|}{ Control-1 } & \multicolumn{2}{|l|}{ Control-2 } & \multicolumn{2}{|c|}{ Water stress-1 } & \multicolumn{2}{|c|}{ Water stress-2 } \\
\hline & Total reads & Unique reads & Total reads & Unique reads & Total reads & Unique reads & Total reads & Unique reads \\
\hline mRNAs & 155,922 & 60,688 & 163,797 & 63,426 & 104,248 & 39,444 & 144,385 & 519,42 \\
\hline miRBase & 415,510 & 1967 & 435,726 & 2019 & 313,001 & 1591 & 430,726 & 1890 \\
\hline Noncoding RNA & 244,891 & 10,514 & 256,746 & 10,703 & 204,979 & 8317 & 274,264 & 9421 \\
\hline Repeats & 463,369 & 28,499 & 490,924 & 29,226 & 325,516 & 22,815 & 451,881 & 26,078 \\
\hline Genome & $1,664,319$ & 416,494 & $1,756,718$ & 436,528 & $1,222,109$ & 333,042 & $1,717,876$ & 453,431 \\
\hline Total & $2,011,156$ & 552,990 & $2,121,444$ & 577,244 & $1,466,922$ & 456,125 & $2,029,346$ & 596,687 \\
\hline
\end{tabular}



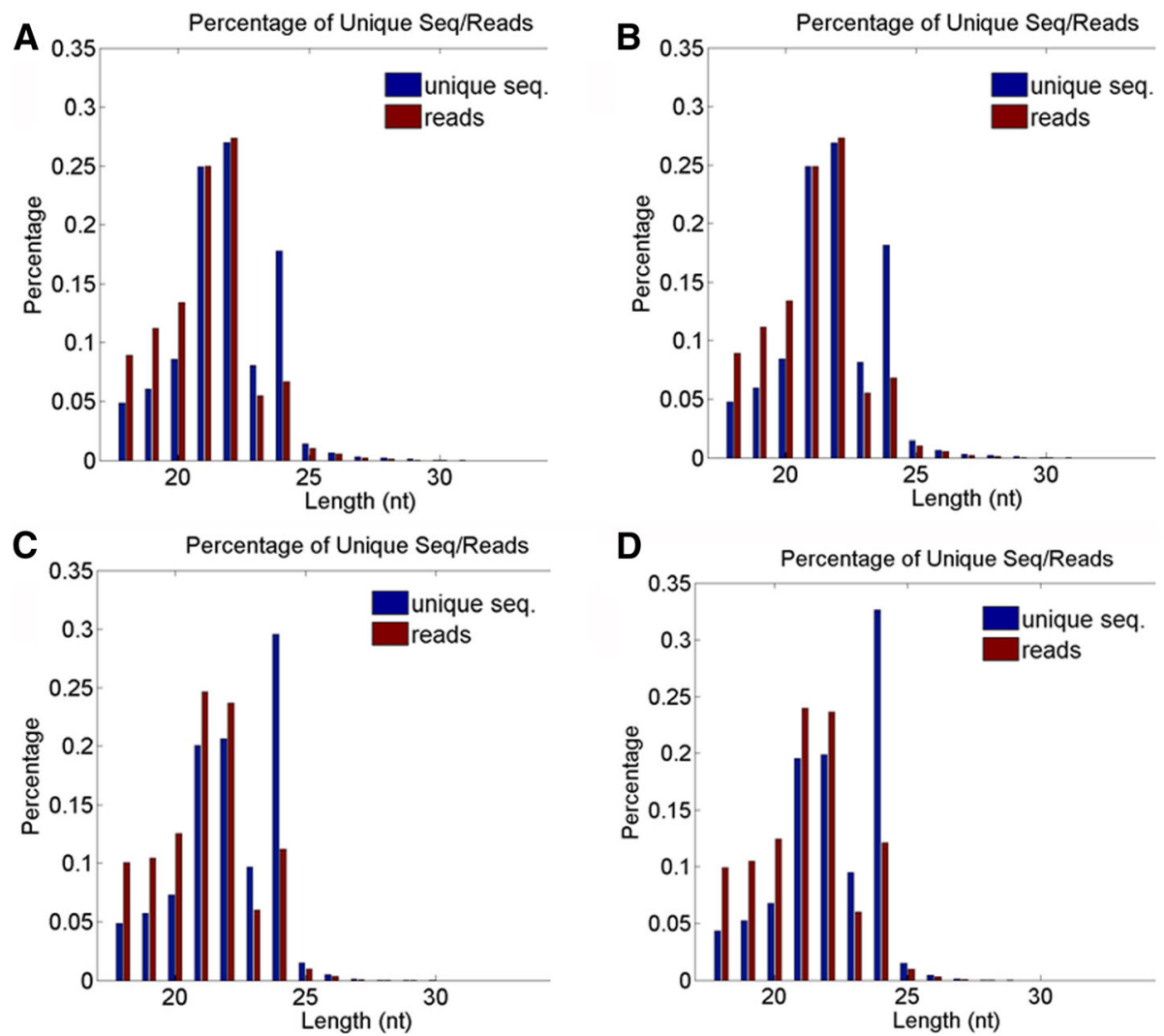

Fig. 1 Total and Unique small RNA length vs abundances was plotted as percent of reads from 4 different small RNA libraries; a and $\mathbf{b}$ are two control libraries (control-1 and control-2) $\mathbf{c}$ and $\mathbf{d}$ are two water-stressed libraries (WS-1 and WS-2)

miRNAs in soybean. Because our classification is strictly based on abundances, this group also included miR395 and miR399, which are highly conserved but expressed at very low levels under normal conditions [20-22].

Among the highly conserved 23 miRNA families [23], two (miR156 and miR166) were classified as high, three (miR168, miR396 and miR172) as moderate and seven (miR169, miR171, miR164, miR390, miR159, miR160 and miR167) as low and two (miR395 and miR399) as extremely low abundantly expressed miRNA families in primary root tips of soybean.

Arikit et al. [7] reported that miR3522 is abundantly expressed in leaf and seed tissues; and miR5770 is enriched in leaf alone. We have identified these two miRNAs in primary root tips (Additional file 2) suggesting that these are ubiquitous in their distribution. It is surprising that this small zone of root tip harbours a diverse population of miRNAs, with 303 miRNAs belonging to 171 families, and several of them are abundantly expressed. These results suggest a critical role for miRNAs in primary root tips, which includes the primary root elongation zone and root apical meristem with actively dividing cells as well as quiescent center (a zone of relatively inactive and slowly dividing cells but can serve as reservoir of stem cells when required) and also the region where vascular differentiation begins. Therefore, it is not surprising to find diverse population of miRNAs that regulates transcription factors as well as other protein coding genes that in turn control multiple developmental processes in the primary root tips.

\section{Differential expression of miRNA families in the primary roots exposed to water deficit}

Most reported plant miRNA regulations in response to stress were based largely on single replicate, whereas we used two replicates. For determining differentially expressed miRNAs during water stress, we used expression differences that are greater than one fold $(\log 2)$ change and multiple test corrected $P$-values of small than 0.05. In total, 23 miRNA families differentially responded to stress, of which 11 families were upregulated while the other 12 were downregulated (Table 2). Among these, miR1512 was highly upregulated (2.71 $\log 2$ fold change) while miR2119 was the most downregulated (4.84 $\log 2$ fold change) miRNA families with higher than 100 RPTM in either control or treatment. Interestingly, both of these most differentially regulated miRNAs represent legume-specific miRNA families. 
Table 2 Differentially expressed miRNA families in soybean primary root tips exposed to water deficit

\begin{tabular}{|c|c|c|c|c|c|c|c|c|}
\hline miRNA_family & Control-1 (RPTM) & Control-2 (RPTM) & Ws-1 (RPTM) & Ws-2 (RPTM) & $\log F C$ & $\log C P M$ & PValue & FDR \\
\hline MIR5037 & 70 & 57 & 0 & 0 & -9.0 & 4.1 & $4.58 \mathrm{E}-14$ & $1.12 \mathrm{E}-12$ \\
\hline MIR2119 & 4192 & 4134 & 157 & 133 & -4.9 & 10.1 & 1.05E-72 & $9.02 \mathrm{E}-71$ \\
\hline MIR5559 & 562 & 434 & 27 & 30 & -4.1 & 7.1 & 1.09E-45 & $6.21 \mathrm{E}-44$ \\
\hline MIR4408 & 45 & 38 & 7 & 5 & -2.8 & 3.7 & 2.11E-04 & $2.12 \mathrm{E}-03$ \\
\hline MIR1536 & 60 & 75 & 14 & 15 & -2.2 & 4.4 & 2.49E-05 & 2.84E-04 \\
\hline MIR403 & 104 & 108 & 34 & 30 & -1.7 & 5.2 & 1.89E-07 & 2.70E-06 \\
\hline MIR171 & 3938 & 3884 & 1138 & 1335 & -1.7 & 10.3 & $4.35 \mathrm{E}-13$ & $9.30 \mathrm{E}-12$ \\
\hline MIR398 & 50 & 57 & 20 & 15 & -1.6 & 4.2 & $2.21 \mathrm{E}-03$ & 1.40E-02 \\
\hline MIR530 & 403 & 434 & 184 & 163 & -1.3 & 7.2 & $2.34 \mathrm{E}-10$ & 3.64E-09 \\
\hline MIR5370 & 94 & 99 & 34 & 49 & -1.2 & 5.2 & 4.08E-04 & 3.49E-03 \\
\hline MIR4397-3 & 139 & 104 & 41 & 74 & -1.1 & 5.5 & $1.68 \mathrm{E}-03$ & 1.15E-02 \\
\hline MIR4391 & 462 & 443 & 259 & 177 & -1.1 & 7.4 & 2.93E-06 & $3.58 \mathrm{E}-05$ \\
\hline MIR3522 & 10,815 & 10,535 & 24,937 & 26,151 & 1.2 & 13.2 & 2.33E-07 & 3.06E-06 \\
\hline MIR408 & 219 & 264 & 504 & 719 & 1.3 & 7.8 & $5.70 \mathrm{E}-12$ & 1.08E-10 \\
\hline MIR4344 & 15 & 19 & 55 & 44 & 1.5 & 4.1 & 5.10E-03 & 3.01E-02 \\
\hline MIR1535 & 30 & 14 & 68 & 79 & 1.7 & 4.6 & 1.55E-04 & 1.66E-03 \\
\hline MIR4411 & 25 & 14 & 82 & 49 & 1.7 & 4.5 & 1.34E-03 & 1.01E-02 \\
\hline MIR167 & 1357 & 1574 & 5426 & 5396 & 1.9 & 10.8 & 6.66E-16 & 1.90E-14 \\
\hline MIR4385 & 10 & 14 & 48 & 44 & 1.9 & 4.0 & 2.02E-03 & $1.33 \mathrm{E}-02$ \\
\hline MIR397 & 254 & 174 & 961 & 917 & 2.1 & 8.2 & $3.29 \mathrm{E}-30$ & $1.41 \mathrm{E}-28$ \\
\hline MIR2111 & 104 & 80 & 382 & 488 & 2.2 & 7.1 & 8.18E-18 & $2.80 \mathrm{E}-16$ \\
\hline MIR1512 & 209 & 207 & 1459 & 1281 & 2.7 & 8.6 & 1.57E-79 & $2.68 \mathrm{E}-77$ \\
\hline MIR4403 & 0 & 0 & 14 & 30 & 7.5 & 2.7 & 3.69E-04 & 3.32E-03 \\
\hline
\end{tabular}

Among the conserved miRNAs, miR403 ( $-1.73 \log 2$ fold change) and miR397 (2.12 log2 fold change) were most differentially regulated families with higher than 100 RPTM in either control or water stress.

Differential regulation of several of these miRNAsin response to water stress was in agreement with previous reports [7-9]. For instance, the observed upregulation of miR397 (stringent criterion) is in agreement with the Kulcheski et al. [9] and downregulation of miR394 (relaxed criterion) (Additional file 2) is similar to the reports of $\mathrm{Li}$ et al. [8]. Interestingly, miR169 regulation differs between drought tolerant and drought sensitive soybean genotypes; it is downregulated in a tolerant genotype while upregulated in a sensitive genotype [8,9]. We used a drought-tolerant soybean cv. Magellan in this study, thus the observed miR169 downregulation (Additional file 2 ) is consistent with the previous reports [9]. Both of these studies $[8,9]$ compared the responses in root system while our study specifically analyzed root tips of seedlings.

It was reported that miR1446 is upregulated under drought stress in soybean leaves [7] but this was not even detected in primary root tips. Another observed difference was with respect to miR3522, which was increased in the primary root tips (Table 2 and Additional file 2) but unaffected in leaves [7] in response to stress. On the other hand, the observed upregulation of miR390 as well as downregulation of miR171 in primary root tips (Table 2 and Additional file 2) is similar to what was observed in leaves of soybean exposed to drought [7]. These similar as well as unique responses suggest that miRNA-mediated target gene regulation under drought is similar as well as distinct in leaves and primary root tips of soybean.

We also compared the abundances of individual miRNAs to identify differentially regulated individual miRNAs (with multiple test corrected $P$-values smaller than 0.05 ), which identified 72 individual miRNAs (Additional file 3). We analyzed the enriched GO terms for 10 of the deregulated individual miRNAs that are highly expressed in either the control or water-stress conditions, and the obtained results were listed in Additional file 4. For example, the targets of an upregulated miRNA, gma-miR156cdeijlm, are enriched in 24 GO terms, such as leaf morphogenesis (GO:0009965) and reproductive developmental process (GO:0003006).

miR-star abundances in primary root tips and their differential regulation in response to water deficit stress The processed miRNA duplex include miRNA that is recruited into the Argonaute-1, which is also more stable 
compared to miR-star, the complementary strand of the duplex. With a few exceptions, the frequency of miR-stars is much lower and largely thought to be non-functional. Consistent with these characteristics, miR-star sequences corresponding to most conserved miRNA families were not recovered from primary root tips (Additional file 1). For those families for which miR-stars were recovered, their abundances were very low compared to their respective miRNA abundances. In the instances where both miRNA and miR-star were sequenced, their normalized abundances did not show any specific ratio. However, some of these miR-star expression levels differed greatly in response to stress, i.e., some were upregulated (miR408a,b,c,d-3p and miR482) while some others were downregulated (miR159d5p, miR171b-5p, miR172b,h,I,j-5p, miR390a,c-3p, miR394a3p, and miR1507c-5p) (Additional file 1).

\section{Novel miRNA identification}

Several deep sequencing efforts have identified many novel miRNAs in soybean [7-9]. Because we used a very specific tissue (primary root tips) in this study, we scrutinized the small RNAs for novel miRNAs, if any. Indeed, on the basis of sequencing both miRNA and miRNAstar sequences [13], we have identified 22 novel miRNAs in the sequenced libraries (Additional file 5). As shown in Fig. 2a, the miRNA and its miRNA* reads were detected for novel miRNAs. Figure $2 \mathrm{~b}$ shows that miRNAs generated from these pre-miRNAs are from either $5^{\prime}$ or $3^{\prime}$ arms of the hairpin structures. Other novel miRNAs in Additional file 4 are similar to the three examples shown in Fig. 2. Only one novel miRNA is significantly downregulated in water-stressed libraries (multiple test corrected $P=0.004$, in Additional file 6).

\section{Discussion}

Prolonged exposure to drought affects plant growth and development, consequently crop yields; even transient droughts can affect plant productivity significantly [24]. Different layers of gene regulatory mechanisms are critical for enduring drought tolerance in plants and

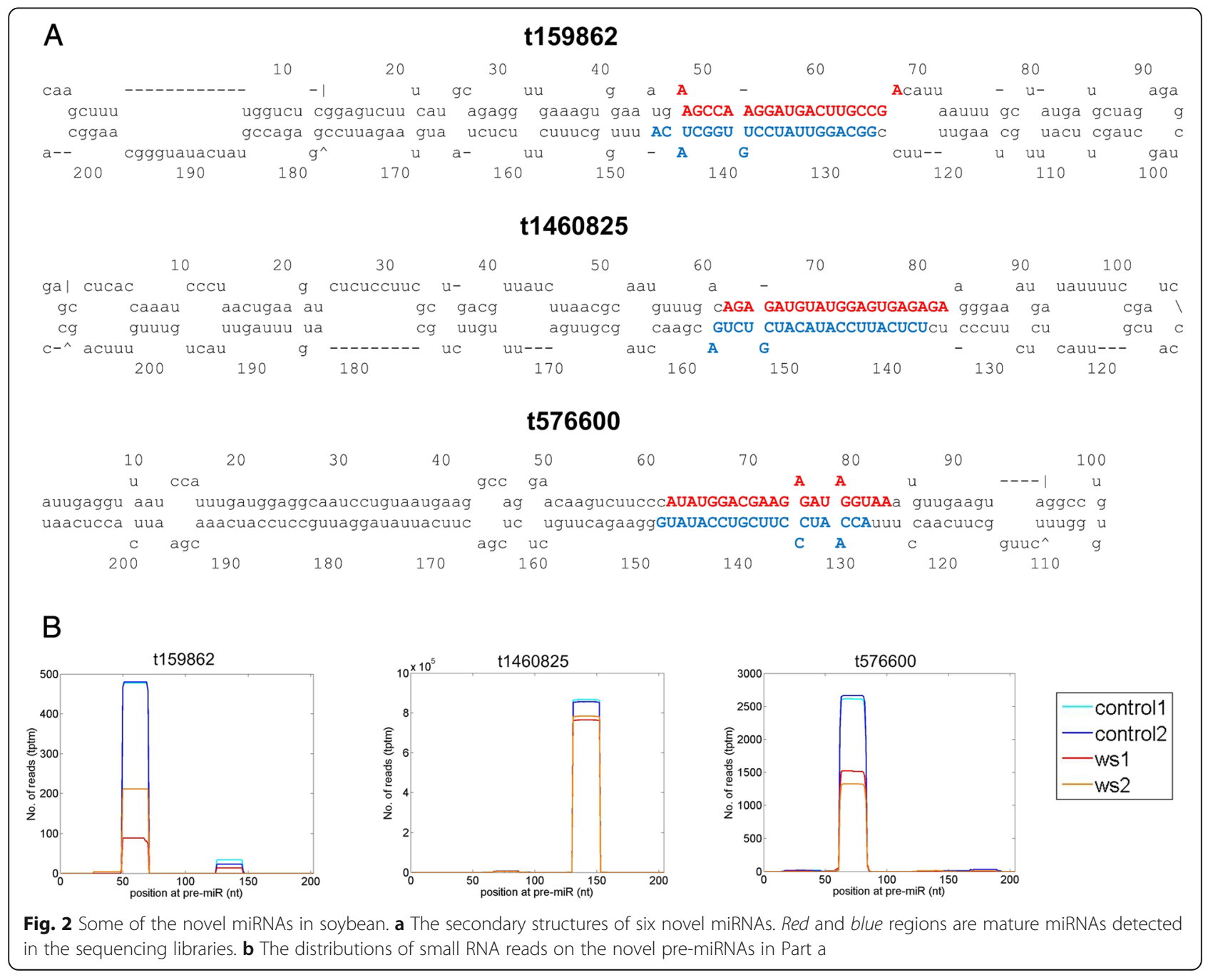


miRNA-dependent post-transcriptional gene regulation is the most recent addition to this list. Primary root tips are critical zone of a root system for water uptake as well as water stress sensing and signalling. We profiled miRNAs in response to water stress in soybean to gain an insight into miRNA-guided gene regulations and its role in stress adaptation. Using stringent criterion, we have identified 12 families (6 families were each up- or down-regulated) as differentially regulated during drought stress (Table 2). However, using relaxed criterion, additional 12 miRNA families were also identified as differentially regulated. Overall, 24 families were found to be differentially expressed in response to water stress in soybean primary root tips (Table 2).

By regulating the expression of SBP transcription factors in leaves, miR156 is known to regulate plant progression into reproductive phase from vegetative phase [25-27]. Besides in leaves, miR156 is abundantly expressed in primary root tips (this study) and the nodules [7] suggesting a much broader role for miR156 family in plants. In different plant species, miR156 is largely upregulated under drought [5]. Most recently, Stief et al. [28] demonstrated that miR156 plays an important role in heat tolerance of Arabidopsis. Even in their study, only 3 variants (MIR156h, d and c) of miR156 family were found to respond to heat suggesting that the overall miR156 regulation would only be marginal and yet sufficient to make Arabidopsis plants to adapt to the recurrent heat by affecting the SBP factors expression [28]. Of the seven miR156 variants we identified in the primary root tips, four are abundantly expressed and were unaffected by the treatment, while two variants that are expressed at low levels (miR156b/f and miR156r) are highly upregulated under stress (Additional file 1). This observation suggests a role for specific variants of miR156 under water stress, probably in specific cell types within the primary root tip.

Being negative regulators of their target mRNAs, downregulation of a miRNA under stress could result in upregulation of the targets and the opposite is true for the upregulated miRNAs [5]. The regulation of transcription factors was studied under water stress conditions in fivecentimeter long root primary roots of soybean [12]. Most importantly, the study found the transcription factors (two CBF-B/NF-YA, a MYB33 gene and TEM1, TEMPRANILLO 1) [7] that are targets of miRNAs were differentially regulated under drought [12]. Our miRNA analysis revealed that the miRNAs targeting above mentioned transcription factors were differentially regulated; miR482 was upregulated while miR169 was downregulated below the stringent threshold level of( $\log 2)$ fold change (Additional file 2). Our results are consistent with the reported upregulation of two NF-YA genes (Glyma02g47380 and Glyma10g10240) [12] that are targeted by miR169 and decreased expression of TEM1 transcription factor [12] that is targeted by miR482. These results demonstrate that the miRNA regulations affected their target genes under stress in the soybean primary root tips.

In soybean primary roots, 37 transcripts associated with auxin synthesis and signaling were regulated by drought [12]. Of these, 17 are auxin response factors that include targets of miR167 (Glyma16g02760.1) and TAS3siRNAs (Glyma06g00950.1), which are downregulated especially under mild and severe stress conditions [12]. The downregulation of these 2 ARFs (Glyma16g02760.1 and Glyma06g00950.1) in primary root tips is consistent with the observed upregulation of miR167, which is induced by almost 2 fold (Table 2). The moderate upregulation of miR390 under water deficit stress could lead to the enhanced accumulation of TAS3 siRNAs as observed in the present study (Additional file 1), which in turn decreases the accumulation of ARFs (ARF2, ARF3 and ARF4). Similarly, the levels of miR393 were also elevated under stress and miR393 targets TIR1, an auxin receptor in plants. The elevated miR393 could decrease TIR1 levels under stress and this will results in decreased ubiquitination of AUX/IAA factors that act as transcriptional repressors. This is consistent with the hypothesis that miRNAs play a critical role in auxin-dependent growth modulation under stress [5]. Overall, three miRNA families that are associated (miR167, miR393 and miR390 and TAS3 siRNAs all displayed either high or moderate upregulation) with auxin sensing and signalling are upregulated in response to water stress. Such a coordinated differential regulation of miRNAs and TAS3siRNAs that target auxin sensing and signaling suggests a more compelling role for these small RNAs under stress. This is somewhat consistent with the fact that the decelerated growth rate in the Region 2 of the primary root tips under drought could be mediated by three miRNAs for conserving water and energy.

miR160 is the only other miRNA family that is known to be involved in auxin signalling but unaltered in response to water stress in this study. In fact, miR160 targets ARF10, ARF16 and ARF17, which have been characterized as transcriptional repressors. Further studies are required to determine whether or not miR160 as well as the other miRNAs that control auxin signaling are differentially regulated under drought in different regions (Region1 and Region 2) of the primary root tips of soybean.

The auxin-mediated gene regulation is dependent on ARFs, but their exact roles as positive or negative regulators of gene expression are largely unknown. On the basis of Arabidopsis ARF promoter:GUS analysis in protoplasts, some have been characterized as activators while some other as repressors [29]. Thus, the overall in vivo role of these ARFs is still unclear in plants. 
In Arabidopsis, $\mathrm{Cu} / \mathrm{Zn}$ SOD upregulation in response to oxidative stress is exclusively dependent on the downregulation of miR398 $[5,20]$. miR398 in plants controls $\mathrm{Cu} / \mathrm{Zn}$ SOD protein abundances not only at the posttranscriptional level by targeting $\mathrm{Cu} / \mathrm{Zn}$ SOD transcripts but also at the posttranslational level by targeting the $\mathrm{CCS}$ gene, that supplies $\mathrm{Cu}$ to the $\mathrm{Cu} / \mathrm{Zn}$ SODs in plants $[5,30]$. Indeed miR398 levels were down-regulated under water stress in primary root tips of soybean (Table 2). These results are consistent with previous reports that the protective proteins associated with the ROS toxicity were upregulated in this region of soybean primary roots exposed to water stress [10].

Recently, several studies reported differential expression of miRNAs such as miR164, miR169, miR171, miR396, miR398, miR399, miR408 and miR2118 in drought-stressed plants but their regulation (up or down) differed between different plant species [5]. Previously, Li et al. [8] analyzed changes in miRNA profiles in roots of a drought-sensitive soybean genotype (inbred line 'HJ-1') while Kulcheski has compared between drought-sensitive 'BR16' with a drought-tolerant 'Embarpa 48'. Li et al. [8] exposed 4 week-old hydroponically grown seedlings to $2 \%$ PEG in $1 x$ Hoagland's solution for $48 \mathrm{~h}$, while Kulcheski et al. [9] studied in 15 day-old seedlings subjected to drought stress by removing the hydroponic solution (similar to that of air drying) for 125 and $150 \mathrm{~min}$. These studies found similar as well as distinct regulations with respect to the responses of various conserved miRNAs. For instance, $\mathrm{Li}$ et al. [8] found that miR166b, miR169d, miR1507a, miR167, miR482, miR4369 and miR4397 were upregulated while miR394 is downregulated. On the other hand Kulcheski et al. [9] have reported that miR166-5p, miR169f-3p, miR1513c, and miR397a,b, were upregulated in sensitive genotype BR16 while these were upregulated in tolerant Embarpa48. Such discrepancies could be due to differences in genotypes, treatments, duration or other variables. Interestingly, miR169 regulation differs between drought tolerant and drought sensitive soybean genotypes; it is downregulated in a tolerant genotype while upregulated in a sensitive genotype $[8,9]$. We used a tolerant soybean $\mathrm{cv}$. Magellan in this study, thus the observed miR169 downregulation is consistent (Additional file 2) with the previous reports [9]. Both of these studies $[8,9]$ compared the responses in an entire root system while our study specifically analyzed root tips of seedlings. Differential responses of miRNAs could be due to several factors such as whether plant species is stress sensitive or tolerant, the tissue that was analyzed, the severity and duration of stress treatment as well as the fact the some of the miRNAs respond transiently while some others show sustained responses to a given stress [31].
To gain an insight into miRNA regulations that are common to diverse abiotic stresses or specific to waterdeficit, we compared our results with $\mathrm{Pi}$ and $\mathrm{N}$ deprivation responses that are available for root comparisons in soybean. Under Pi deficiency downregulation of miR169 and miR159 whereas upregulation of miR482 and miR397 was reported [32] and similar responses were found during water deficit (Table 2 and Additional file 2). However, miR3522 differed in its response that it was downregulated under Pi deficiency [32] while it was upregulated in our study. Another study [33] reported upregulation of miR159 under Pi deficiency in soybean roots, which contradicts with the results from $\mathrm{Xu}$ et al. [32] as well as our analysis under water deficit stress (Additional file 2 and Table 2). In response to $\mathrm{N}$ deprivation, down regulation of miR171 and miR398 [34] was reported, which is in agreement with our analysis under water stress. On the other hand, miR166 and miR390 was downregulated by $\mathrm{N}$ deficiency [34] whereas these were upregulated under water stress (Additional file 2 and Table 2). Overall it appears that there are similarities and differences with respect to miRNA regulations under water stress and nutrient deprivations. However, it should be noted that our study used primary root tips while those compared studies used entire root system, so these comparisons need to be cautiously interpreted.

\section{Conclusions}

Both root elongation and differentiation processes are important aspects of how plants respond to a variety of environmental factors that limit growth. Characterization of roles of miRNAs in stress regulatory networks in the primary root tips would help better understand the mechanisms of stress tolerance as well as provide new opportunities to engineer plant's drought tolerance. Yamaguchi et al., [10] had investigated root growth adaptation to low water potential in soybean, specifically with respect to the protein alterations in the elongation zone of the primary root tip (region-specific analysis). To maintain root elongation under water deficit, two potential mechanisms have been suggested: osmotic adjustment and modification of $\mathrm{CW}$ extensibility [35]. Osmotic adjustment mostly involves net accumulation of osmolytes such as sugars (fructose, glucose, and sucrose), amino acids (mostly proline) and glycine betaine $[24,36]$. Modification of cell wall extensibility mostly includes protein synthesis and modification of carbohydrates. None of the differentially regulated miRNAs can be directly linked with such processes. However, it is worth noting that TFs targeted by miRNAs could be regulating many genes that control these two major processes, thus, miRNAs are likely to play an indirect role in modulating drought responses in the primary root tips. 


\section{Additional files}

Additional file 1: Table S1. The normalized abundances of individual conserved miRNAs. (XLSX $46 \mathrm{~kb}$ )

Additional file 2: Table S2. The normalized abundances of miRNA families in control and water-stressed roots tips of soybean. (XLSX $36.2 \mathrm{~kb}$ )

Additional file 3: Table S3. The deregulated miRNAs in water-stress condition. (XLSX $19 \mathrm{~kb}$ )

Additional file 4: Table S4. The enriched GO terms of predicted targets for ten deregulated miRNAs in water-stress conditions. (XLSX $149 \mathrm{~kb})$

Additional file 5: Table S5. The novel miRNAs identified in soybean. (XLSX $14 \mathrm{~kb}$ )

Additional file 6: Table S6. The normalized abundances of novel miRNAs. (XLSX 13 kb)

\section{Acknowledgements}

The research was supported in part by Oklahoma Agricultural experiment station to RS, a grant (No. 31460295) of National Natural Science Foundation of China (http://www.nsfc.gov.cn/) and a grant (No. SKLGE-1511) of the Open Research Funds of the State Key Laboratory of Genetic Engineering, Fudan University, China, to YZ, and funding support from the Missouri Soybean Merchandising Council project (No. 220) to HTN. We acknowledge Dr. Robert E. Sharp, University of Missouri, for providing access to the isopiestic thermocouple psychrometer and the growth chamber facility for the root tip tissue collection and related discussions. VH acknowledge a postdoctoral fellowship from the University Grants Commission, Government of India.

\section{Declaration}

This article has been published as part of BMC Systems Biology Volume 10 Supplement 5, 2016. 15th International Conference On Bioinformatics (INCOB 2016): systems biology. The full contents of the supplement are available online http://bmcsystbiol.biomedcentral.com/articles/supplements/volume10-supplement-5

\section{Funding}

Publication of this article was funded by the Oklahoma Agricultural Experiment Station.

\section{Availability of data and materials}

The obtained small RNA profiles of this study were available at the NCBI SRA database under the series accession number SRP083572.

\section{Authors' contributions}

BV, MC, HTN planned and performed the experiments, CL-B, AF, GM, generated and sequenced the libraries, $Y Z, X Z, R S$ and $V H$ analyzed the sequences, and $\mathrm{VH}, \mathrm{YZ}$ and $\mathrm{RS}$ wrote the manuscript. All authors checked and approved the final manuscript.

\section{Competing interests}

The authors declare that they have no competing interests.

\section{Consent for publication}

Not applicable.

\section{Ethics approval and consent to participate}

Not applicable.

\section{Author details}

${ }^{1}$ Faculty of Information Engineering and Automation, Kunming University of Science and Technology, Kunming, Yunnan 650500, China. ${ }^{2}$ Department of Biochemistry and Molecular Biology, Oklahoma State University, Stillwater, OK 74078, USA. ${ }^{3}$ National Center for Soybean Biotechnology and Division of Plant Sciences, University of Missouri, Columbia, MO 65211, USA. ${ }^{4}$ Institut of Plant Sciences Paris-Saclay (IPS2), CNRS, INRA, University of "Paris-Sud", Batiment 630, 91405 Orsay, France. ${ }^{5}$ Institut of Plant Sciences Paris-Saclay (IPS2), CNRS, INRA, University of "Paris-Diderot", Sorbonne Paris-Cité, 91405
Orsay „, Paris, France. ${ }^{6}$ National Center for Genome Resources, Santa Fe, New Mexico, NM 87505, USA. 'Present address: Pioneer Hi-Bred International, Inc Johnston, IA 50131, USA

Published: 5 December 2016

\section{References}

1. Sunkar R, Zhu JK. Micro RNAs and short-interfering RNAs in plants. J Integr Plant Biol. 2007:49:817-26.

2. Chen X. Small RNAs and their roles in plant development. Annu Rev Cell Dev. 2009:25:21-44.

3. Jones-Rhoades MW, Bartel DP, Bartel B. MicroRNAs and their regulatory roles in plants. Annu Rev Plant Biol. 2006:57:19-53.

4. Mallory AC, Vaucheret $\mathrm{H}$. Functions of microRNAs and related small RNAs in plants. Nat Genet. 2006:38:S31-6.

5. Sunkar R, Li Y-F, Jagadeeswaran G. Functions of microRNAs in plant stress responses. Trends Plant Sci. 2012;17:196-203.

6. Deshmukh R, Sonah H, Patil G, Chen W, Prince S, Mutava R, Vuong V, Valliyodan B, Nguyen HT. Integrating omic approaches for abiotic stress tolerance in soybean. Front Plant Sci. 2014:5:244.

7. Arikit S, Xia R, Kakrana A, Huang K, Zhai J, Yan Z, Valdés-ópez O, Prince $\mathrm{S}$, Musket TA, Nguyen HT, et al. An atlas of soybean small RNAs identifies phased siRNAs from hundreds of coding genes. Plant Cell. 2014:26:4584-601

8. Li H, Dong Y, Yin H, Wang N, Yang J, Liu X, Wang Y, Wu J, Li X. Charactrization of the stress associated microRNAs in Glycine max by deep sequencing. BMC Plant Biol. 2011;11:170.

9. Kulcheski FR, de Oliveira LF, Molina LG, Almerão MP, Rodrigues FA, Marcolino J, et al. Identification of novel soybean microRNAs involved in abiotic and biotic stresses. BMC Genomics. 2011;12:307.

10. Yamaguchi M, Valliyodan B, Zhang J, Lenoble ME, Yu O, Rogers EE, Nguyen $H T$, Sharp RE. Regulation of growth response to water stress in the soybean primary root I. Proteomic analysis reveals region-specific regulation of phenylpropanoid metabolism and control of free iron in the elongation zone. Plant Cell Environ. 2010:33:223-43.

11. Yamaguchi M, Sharp RE. Complexity and coordination of root growth at low water potentials: recent advances from transcriptomic and proteomic analyses. Plant Cell Environ. 2010;33:590-603.

12. Song L, Prince S, Valliyodan B, Joshi T, Maldonado dos Santos JV, Wang J, Lin L, Wan J, Wang Y, Xu D, Nguyen HT. Genome-wide transcriptome analysis of soybean primary root under varying water-deficit conditions. BMC Genomics. 2016:17:57.

13. Meyers BC, Axtell MJ, Bartel B, Bartel DP, Baulcombe D, Bowman JL, Cao X Carrington JC, Chen X, Green PJ, et al. Criteria for annotation of plant microRNAs. Plant Cell. 2008;20:3186-90

14. Robinson MD, McCarthy DJ, Smyth GK. edgeR: a Bioconductor package for differential expression analysis of digital gene expression data. Bioinformatics. 2010:26:139-40.

15. Zheng $Y$, Zhang W. Animal microRNA target prediction using diverse sequence-specific determinants. J Bioinform Comput Biol. 2010;8:763-88.

16. Du Z, Zhou X, Ling Y, Zhang Z, Su Z. agriGO: a GO analysis toolkit for the agricultural community. Nucleic Acids Res. 2010;38 suppl 2:W64-70.

17. Debernardi JM, Mecchia MA, Vercruyssen L, Smaczniak C, Kaufmann K, Inze D, Rodriguez RE, Palatnik JF. Post-transcriptional control of GRF transcription factors by microRNA miR396 and GIF co-activator affects leaf size and longevity. Plant J. 2014;79:413-26.

18. Omidbakshshfard MA, Proost S, Fujikama U, Mueller-Roeber B. GrowthRegulating Factors (GRFs): a small transcription factor family with important functions in plant biology. Mol Plant. 2015:8:998-1010.

19. Fei $\mathrm{Q}$, Xia R, Meyers BC. Phased, secondary, small interfering RNAs in posttranscriptional regulatory networks. Plant Cell. 2013;25:2400-15.

20. Jagadeeswaran G, Saini A, Sunkar R. Biotic and abiotic stress down-regulate miR398 expression in Arabidopsis. Planta. 2009;229:1009-14.

21. Li Z, Zheng Y, Jagadeeswaran G, Li Y, Gowdu K, et al. Identification and temporal expression analysis of conserved and novel miRNAs in Sorghum. Genomics. 2011:98:460-8.

22. Li Y, Zheng Y, Jagadeeswaran G, Sunkar R. Characterization of small RNAs and their target genes in wheat seedlings using sequencing-based approaches. Plant Sci. 2013;203:17-24

23. Sunkar R, Jagadeeswaran G. In silico identification of conserved miRNAs in large number of diverse plant species. BMC Plant Biol. 2008;8:37. 
24. Bartels D, Sunkar R. Drought and salt tolerance in plants. Crit Rev Plant Sci. 2005;24:23-58.

25. Matts J, Roe BA, Sunkar R. Identification of microRNAs and their targets in switchgrass, a model cellulosic biofuel plant species. J Plant Physiol. 2010;167(11):896-904.

26. Fu C, Sunkar R, Zhou C, Shen H, Zhang J, Matts J, Wolf J, Mann DGJ, Neat Stewart C, Tang Y, Wang Z-Y. Overexpression of miR156 in switchgrass results in various morphological alterations and leads to improved biomass production. Plant Biotechnol J. 2012;10:443-52.

27. Poethig RS. Small RNAs and developmental timing in plants. Curr Opin Genet Dev. 2009:19:374-8.

28. Stief P, Fuchs-Ocklenburg S, Kamp A, Manohar CS, Houbraken J, Boekhout T, et al. Dissimilatory nitrate reduction by Aspergillusterreus isolated from the seasonal oxygen minimum zone in the Arabian Sea. BMC Microbiol. 2014;14:35.

29. Tiwari SB, Hagen G, Guilfoyle T. The roles of auxin response factor domains in auxin-responsive transcription. Plant Cell. 2013:15:533-43.

30. Li Y, Zheng Y, Addo-quaye C, Li Z, Saini A, Jagadeeswaran G, Axtell M, Zhang W, Sunkar R. Transcriptome-wide identification of microRNA targets in rice. Plant J. 2010;62:742-59.

31. Sunkar R. MicroRNAs with macro effects on plant stress responses. Semin Cell Dev Biol. 2010;21(8):805-81.

32. Xu F, Liu Q, Chen L, Kuang J, Walk T, Wang J, Liao H. Genome-wide identification of soybean microRNAs and their targets reveals their organspecificity and responses to phosphate starvation. BMC Genomics. 2013;14:66.

33. Zeng $H Q$, Zhu $Y Y$, Huang $S Q$, Yang ZM. Analysis of phosphorus-deficient responsive miRNAs and cis-elements from soybean (Glycine max L.). J Plant Physiol. 2010;167:1289-97.

34. Wang Y, Zhang C, Hao Q, Sha A, Zhou R, Zhou X, Yuan L. Elucidation of miRNA-mediated responses to low nitrogen stress by deep sequencing of two spybean genotypes. PLoS One. 2013;8:e67423.

35. Sharp RE, Poroyko V, Hejlek LG, Spollen WG, Springer GK, Bohnert HJ, Nguyen HT. Root growth maintenance during water deficits: physiology to functional genomics. J Exp Bot. 2004;55:2343-51.

36. Morgan JM. Osmoregulation and water stress in higher plants. Annu Rev Plant Physiol. 1984;35:299-319.

\section{Submit your next manuscript to BioMed Central and we will help you at every step:}

- We accept pre-submission inquiries

- Our selector tool helps you to find the most relevant journal

- We provide round the clock customer support

- Convenient online submission

- Thorough peer review

- Inclusion in PubMed and all major indexing services

- Maximum visibility for your research

Submit your manuscript at www.biomedcentral.com/submit

C) Biomed Central 